\section{BAS/ CIRCULATING CHEMICAL AND CELLULAR INJURY/REPAIR BSCR39 RESPONSES ARE LINKED TO CARDIAC DYSFUNCTION AND REMODELLING IN HUMAN MYOCARDIAL INFARCTION}

doi:10.1136/hrt.2010.205781.50

${ }^{1,2} \mathrm{R}$ J McGeoch, ${ }^{1} \mathrm{~A}$ McGarrity, ${ }^{1,2} \mathrm{~A}$ R Payne, ${ }^{2} \mathrm{~S}$ Watkins, ${ }^{2} \mathrm{~T}$ Steedman, ${ }^{2} \mathrm{~A}$ Davie, ${ }^{2} \mathrm{~W} S$ Hillis, ${ }^{2} \mathrm{M}$ M Lindsay, ${ }^{2} \mathrm{~S} D$ Robb, ${ }^{3} \mathrm{E}$ Rhaume, ${ }^{3} \mathrm{~J} \mathrm{C}$ Tardif, ${ }^{2} \mathrm{~K}$ G Oldroyd, ${ }^{1,2} \mathrm{C}$ Berry. ${ }^{1} \mathrm{BHF}$ Glasgow Cardiovascular Research Centre, University of Glasgow, Glasgow, Scotland, UK; ${ }^{2}$ Golden Jubilee National Hospital, Clydebank, UK; ${ }^{3}$ Montreal Heart Institute, Montreal, Canada

Background Endogenous regenerative pathways may contribute to cardiovascular repair following ischaemic injury. Based on recent results in experimental studies, we investigated candidate endogenous chemical and cellular injury/repair responses in human myocardial infarction (MI)

Methods Circulating injury (eg, platelets) and repair (circulating CD34+ progenitor cells, serum vascular endothelial growth factor (VEGF) and thymosin $\beta 4$, and urine acetyl-Ser-Asp-Lys-Pro $(\mathrm{AcSDKP})$ ) responses were quantified 2 days and 3 months after acute MI. Progenitor cells in whole blood were quantified using flow cytometry, and cytokines were measured by immunoassay. An automated analyser was used for haematological measurements. Invasive measures of coronary artery microvascular resistance and collateral supply were measured acutely using coronary thermodilution techniques. Cardiac function and remodelling were quantified by magnetic resonance imaging.

Results 35 consecutive patients with MI (mean \pm SD age $58 \% \pm$ 10 years) were included. AcSDKP measured 2 days post-MI negatively predicted left ventricular ejection fraction $\left(R^{2}=0.43 ; p=0.024\right)$ and positively predicted left ventricular end-systolic volume index $\left(\mathrm{R}^{2}=0.56 ; \mathrm{p}=0.011\right)$ at 3 months. At follow-up, CD34+ count negatively predicted myocardial infarct mass $\left(R^{2}=0.29 ; p=0.015\right)$ and left ventricular end-systolic volume index $\left(R^{2}=0.20 ; p=0.02\right)$. In multivariable analyses, haemoglobin concentration measured 2 days post-MI negatively predicted coronary collateral supply $(\mathrm{p}=0.006)$, whereas red cell distribution width $(p=0.004)$ and platelet count $(p=0.0001)$ positively predicted coronary collateral supply. Serum VEGF at 3 months and change in VEGF were negative multivariable predictors of left ventricular end-diastolic volume index at 3 months ( $p=0.021$ and $p=0.006$, respectively).

Conclusion Circulating chemical and cellular responses participate in myocardial injury and repair and represent targets for therapeutic development.

Acknowledgements We acknowledge the support of the British Heart Foundation and the Chief Scientist Office.

\section{BAS/ CORONARY HEART DISEASE RISK ASSOCIATED WITH THE BSCR40 HOMOZYGOUS MINOR ALLELE FOR ENDOTHELIAL PROTEIN C RECEPTOR SER219GLY}

doi:10.1136/hrt.2010.205781.51

${ }^{1} \mathrm{H}$ A Ireland, ${ }^{1} \mathrm{~J}$ A Cooper, ${ }^{1} \mathrm{P} \mathrm{J}$ Talmud, ${ }^{2} \mathrm{~A} \mathrm{D}$ Hingorani, ${ }^{2} \mathrm{M}$ Kivimki, ${ }^{2} \mathrm{M}$ Kumari, ${ }^{3} \mathrm{R}$ Morris, ${ }^{4} \mathrm{~T}$ Tzoulaki, ${ }^{5} \mathrm{~J}$ Price, ${ }^{5} \mathrm{~F}$ G Fowkes, ${ }^{1} \mathrm{~S}$ E Humphries. ${ }^{1}$ Division of Medicine, UCL, London, UK; ${ }^{2}$ Division of Epidemiology and Public Health, UCL, London, UK; ${ }^{3}$ Division of Primary Care and Population Health, Royal Free Campus, UCL, London, UK; ${ }^{4}$ Department of Epidemiology and Biostatistics, Imperial College London; London, UK; ${ }^{5}$ Centre for Population Health Sciences, University of Edinburgh, Edinburgh, UK

Rationale for the study Endothelial protein C receptor (EPCR) is involved in the regulation of thrombin generation and inflammation. PROCR gene variant rs867186 codes for an amino acid substitution (Ser219Gly) within the membrane spanning region of EPCR. The minor allele of the variant has been identified as functional, and associated with increased shedding of EPCR from the endothelial surface. ${ }^{1}$ Previous analysis has also shown increased coagulation activated markers in those with the Gly allele1. ${ }^{2}$ Furthermore, while heterozygotes for this variant may be protected from coronary heart disease (CHD), homozygotes had a threefold elevation in $\mathrm{CHD}$ risk in a prospective cohort (NPHSII). ${ }^{1}$ In a case-control study of CHD (HIFMECH), there was a suggestion that the EPCR genotype interacts with factors present in metabolic syndrome, to increase CHD risk. ${ }^{1}$

Methodology NPHSII was analysed to further assess the CHD risk associated with EPCR Ser219Gly in those who have been identified as diabetic, or as having 'metabolic syndrome', during 15 years' follow-up.

Results Individuals who were Gly/Gly and had 'metabolic syndrome' had a fourfold increase in CHD risk compared with Ser/ Ser $(H R=4.72$, CI 1.69 to 13.4) $p=0.006$; or an eightfold increase in risk above those who were Ser/Ser without metabolic syndrome $(\mathrm{HR}=8.02$, CI 2.89 to 22.2) $\mathrm{p}=0.006$. No Gly/Gly individuals without metabolic syndrome had a CHD event.

Conclusions Homozygotes for the Gly allele are present at a frequency of $\sim 1 \%$. If the findings in the study can be replicated, the variant would constitute a considerable CHD risk in $1 \%$ of diabetics. Replication studies are under way in three further, large prospective cohorts.

\section{REFERENCES}

1. Ireland H, Konstantoulas CJ, Cooper JA. EPCR Ser219Gly: Elevated sEPCR, prothrombin $\mathrm{F} 1+2$, risk for coronary heart disease, and increased SEPCR shedding in vitro. Atherosclerosis 2005; 183:283-89.

2. Ireland HA, Cooper JA, Drenos F, et al. FVII, FVIla, and downstream markers of extrinsic pathway activation differ by EPCR Ser219Gly variant in healthy men. Arterioscler Thromb Vasc Biol 2009;29:1968-74.

\section{BAS/ MODULATION OF EXTRACELLULAR MATRIX PROTEIN BSCR41 EXPRESSION BY INTERLEUKIN 1 IN HUMAN CARDIAC MYOFIBROBLASTS: REGULATION BY P38 MAP KINASE}

doi:10.1136/hrt.2010.205781.52

N A Turner, P Warburton, K E Porter. Division of Cardiovascular and Neuronal Remodelling, Multidisciplinary Cardiovascular Research Centre (MCRC), University of Leeds, Leeds, UK

The proinflammatory cytokine interleukin 1 (IL-1) elicits catabolic effects on the myocardial extracellular matrix (ECM) early after myocardial infarction but there is little understanding of its direct effects on cardiac myofibroblasts (CMF), a key cell type involved in the regulation of myocardial remodelling. We used a focused RTPCR microarray to investigate the effects of IL- 1 on expression of 41 ECM genes in CMF cultured from different patients, and explored the regulatory role of the p38 MAPK signalling pathway. IL-1 $(10 \mathrm{ng} / \mathrm{ml}, 6 \mathrm{~h})$ had only a minimal effect on mRNA expression of structural ECM proteins, including collagens, laminins, fibronectin and vitronectin. However, IL-1 induced marked increases in expression of several ECM proteases, including matrix metalloproteinases MMP-1 (collagenase-1), MMP-3 (stromelysin-1), MMP-9 (gelatinase-B) and MMP-10 (stromelysin-2). Conversely, IL-1 reduced mRNA expression of ADAMTS-1, a metalloproteinase that suppresses neovascularisation. IL-1 stimulated a small increase in expression of tissue inhibitor of metalloproteinases (TIMP)-1, but not TIMP-2. Data for MMPs 1, 2, 3, 9 and 10 and ADAMTS- 1 were confirmed by quantitative real-time RT-PCR. IL- 1 strongly activated the p38 MAPK pathway in human CMF, as determined by immunoblotting with phospho-specific antibodies. A p38 MAPK inhibitor (SB203580) reduced IL-1-induced mRNA expression of MMP-3, but did not affect expression of any of the other MMPs studied. SB203580 also markedly reduced ADAMTS-1 mRNA expression.

In summary, IL-1 induces a distinct pattern of ECM protease expression in human CMF, in part regulated by p38 MAPK, 